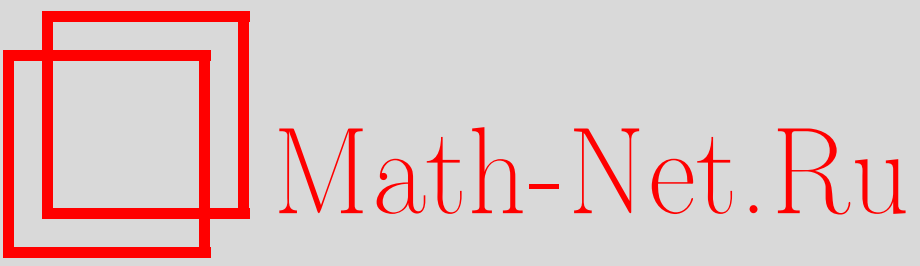

Р. Де Лео, Существование и мера эргодических слоений в задаче Новикова о полуклассическом движении электрона, УМН, 2000, том 55, выпуск 1, 181-182

DOI: https://doi.org/10.4213/rm252

Использование Общероссийского математического портала Math-Net.Ru подразумевает, что вы прочитали и согласны с пользовательским соглашением

http://www . mathnet.ru/rus/agreement

Параметры загрузки:

IP: 54.84 .234 .179

26 апреля 2023 г., 17:52:18 


\title{
СУЩЕСТВОВАНИЕ И МЕРА ЭРГОДИЧЕСКИХ СЛОЕНИЙ В ЗАДАЧЕ НОВИКОВА О ПОЛУКЛАССИЧЕСКОМ ДВИЖЕНИИ ЭЛЕКТРОНА
}

\author{
Роьерто ДЕ ЛЕо
}

Задача о полуклассическом движении электрона в решетке под действием сильного магнитного поля приводит к очень интересной задаче маломерной "периодической" топологии [1], а именно, изучению плоских сечений двумерных поверхностей в $\mathbb{T}^{3}$. В почти рациональном случае А.В. Зорич [2] и в случае общего положения И. А. Дынников [3] доказали гипотезу Новикова, а именно, что открытые орбиты имеют сильное асимптотическое направление; на основе проведенного ими анализа С. П. Новиков [4] извлек следующую замечательную картину: при фиксированном законе дисперсии каждому направлению магнитного поля, порождающему открытые слои общего положения, соответствует единственный неделимый целочисленный 2 -цикл в $\mathbb{T}^{3}$, который локально постоянен.

Это означает, что на двумерной сфере задано счетное семейство попарно непересекающихся открытых областей, занумерованных взаимно простыми тройками целых чисел. Объединение этих зон есть открытое всюду плотное подмножество сферы, но пока не ясно, имеет оно полную меру или нет. Во всяком случае, при фиксированной энергии это множество вместе с множеством направлений, для которых все траектории замкнуты, имеет полную меру (см. ниже).

С.П. Новиков и А.Я. Мальцев [5] применили эти результаты к теории проводимости в нормальных металлах, описав новые универсальные топологические явления, наблюдаемые экспериментально. Поэтому очень важно, в том числе с физической точки зрения, изучить подробнее неизбежно появляющиеся направления магнитного поля необщего положения.

Ниже мы приводим набор наиболее сильных на сегодняшний день утверждений о мере и свойствах указанных зон. Эти утверждения нужны для физических приложений. Они основаны на технике, развитой И.А. Дынниковым, и не были сформулированы в более ранних публикациях. Во время подготовки к печати данной работы И. А. Дынников сообщил мне, что эти результаты опубликованы в его недавней работе [6].

Лемма. Для закона дисперсии общего положения $f \in C^{\infty}\left(\mathbb{T}^{3}\right)$ границы зон устойчивости являются кусочно гладкими, и две различные зоны соприкасаются лиив $в$ счетном числе точек.

СлЕДСтвИЕ. Для закона дисперсии общего положения $f \in C^{\infty}\left(\mathbb{T}^{3}\right)$ либо имеется всего одна зона устойчивости, которая покрывает всю сферу, либо их бесконечно много, и тогда найдется бесконечное чилсо "эргодических" направлений магнитного поля.

Теорема 1. Для любого $f \in C^{\infty}\left(\mathbb{T}^{3}\right)$ множество направлений, порождающих “эргодические" слои, имеет нулевую меру для почти всех значений функции $f$.

Теорема 2. Для функции $f \in C^{\infty}\left(\mathbb{T}^{3}\right)$ общего положения множсество "әргодических" направлений имеет нулевую меру для любого фиксированного значения функиии $f$.

Доказательство следствия основано на том, что любой путь общего положения на $\mathbb{S}^{2}$ пересекает множество особых направлений по канторовскому множеству, поэтому множество этих направлений образует фрактальную структуру на сфере.

Аналитическое исследование свойств этого фрактала оказывается очень сложной задачей. Из теорем 1 и 2 следует, что для ориентированной поверхности общего положения множество особых направлений имеет меру нуль, но до сих пор открыт вопрос о том, останется ли нулевой мера множества "эргодических" направлений, если мы рассмотрим целый интервал уровней энергии, что имеет отношение к [5].

С.П. Новиков выдвинул гипотезу, что в случае общего положения множество "эргодических" направлений для фиксированной энергии имеет на сфере фрактальную размерность $\alpha \in(0,1)$, а множество всех таких направлений имеет фрактальную размерность $\beta \in(1,2)$.

Автором были выполнены вычисления [7] для функции $f(x, y, z)=\cos (x)+\cos (y)+\cos (z)$, для которой ввиду симметрии $f\left(x^{\alpha}+\pi\right)=-f\left(x^{\alpha}\right)$ все зоны устойчивости имеют наибольший размер при $E=0$.

Вычисления проводились следующим образом. Поскольку поверхность $M^{2}=f^{-1}(0)$ имеет род 3 и соответствующее вложение $i: M^{2} \rightarrow \mathbb{T}^{3}$ имеет ранг 3 , мы можем найти канонический 
базис циклов $\left\{e_{j}, f_{j}\right\}$ на $M^{2}$ такой, что $i_{*}\left(e_{j}\right)=0$ и $i_{*}\left(f_{j}\right)$ составляют базис в $H_{1}\left(\mathbb{T}^{3}, \mathbb{Z}\right)$. Программа перебирает все рациональные значения магнитного поля $H=(m / N, n / N, 1), N \geqslant n \geqslant$ $m \geqslant 0$, для некоторого фиксированного $N$, для всех них находит критические точки и вычисляет гомологический класс соответствуюшей петли в $\mathbb{T}^{3}$ и ее индексы пересечений в $M^{2}$ с циклами $i_{*}\left(f_{j}\right)$.

Как только найдена одна из критических петель, гомологичная нулю в $\mathbb{T}^{3}$, ее индексы пересечения автоматически дают гомологический класс неделимого 2 -цикла $l \in H_{2}\left(\mathbb{T}^{3}, \mathbb{Z}\right)$, который отвечает данному магнитному полю.

В следующей таблице даны результаты вычислений, полученные при $N=400$ :

\begin{tabular}{|c|c|c|c|}
\hline Класс гомологий & Площадь & Класс гомологий & Площадь \\
\hline$(0,0,1)$ & $(2.83 \pm .02) 10^{-1}$ & $(1,5,5)$ & $(4.1 \pm .2) 10^{-3}$ \\
\hline$(1,1,1)$ & $(2.03 \pm .01) 10^{-1}$ & $(2,5,8)$ & $(4.1 \pm .4) 10^{-3}$ \\
\hline$(1,2,2)$ & $(8.2 \pm .2) 10^{-2}$ & $(2,6,7)$ & $(3.4 \pm .4) 10^{-3}$ \\
\hline$(0,1,2)$ & $(5.1 \pm .1) 10^{-2}$ & $(4,7,8)$ & $(3.0 \pm .3) 10^{-3}$ \\
\hline$(1,3,3)$ & $(2.1 \pm .1) 10^{-2}$ & $(0,3,4)$ & $(2.9 \pm .4) 10^{-3}$ \\
\hline$(2,3,4)$ & $(1.7 \pm .1) 10^{-2}$ & $(3,5,7)$ & $(2.7 \pm .3) 10^{-3}$ \\
\hline$(1,3,5)$ & $(9.6 \pm .5) 10^{-3}$ & $(1,6,6)$ & $(2.0 \pm .1) 10^{-3}$ \\
\hline$(1,4,6)$ & $(9.6 \pm .5) 10^{-3}$ & $(4,5,8)$ & $(2.0 \pm .4) 10^{-3}$ \\
\hline$(0,2,3)$ & $(9.0 \pm .6) 10^{-3}$ & $(5,8,10)$ & $(1.9 \pm .4) 10^{-3}$ \\
\hline$(2,4,5)$ & $(8.6 \pm .6) 10^{-3}$ & $(4,6,9)$ & $(1.8 \pm .3) 10^{-3}$ \\
\hline$(1,4,4)$ & $(8.3 \pm .3) 10^{-3}$ & $(1,6,10)$ & $(1.7 \pm .1) 10^{-3}$ \\
\hline$(1,2,4)$ & $(6.2 \pm .5) 10^{-3}$ & $(5,9,11)$ & $(1.6 \pm .2) 10^{-3}$ \\
\hline$(3,4,6)$ & $(4.7 \pm .5) 10^{-3}$ & $(4,6,7)$ & $(1.5 \pm .2) 10^{-3}$ \\
\hline
\end{tabular}

Исползуя метод "Вox Counting" из [8] для полученных данных, т.е. находя число зон $A_{k}$ с плошадью между $n^{k}$ и $n^{k+1}$ для некоторого фиксированного $n$ и интерполируя последовательность $\left\{A_{k}\right\}$ линейным образом, можно найти грубую оценку для фрактальной размерности дополнительного множества, т.е. множества особых направлений.

Из данных, полученных при $N=400$, мы извлекли такую оценку для фрактальной размерности эргодических режимов: $\beta \simeq 1.2$, которая согласуется с гипотезой Новикова. Дальнейшие вычисления при $N=1000$, которые, возможно, дадут лучшую оценку, будут проделаны в ближайшее время.

Автор благодарен С. П. Новикову за постановку задачи и многочисленные полезные указания, а также И.А. Дынникову и А. Джакоббе за многочисленные полезные обсуждения. Автор благодарит также Indam за финансовую поддержку.

\section{СПИСОК ЛИТЕРАТУРЫ}

[1] Новиков С. П. // УМН. 1982. Т. 37. №5. С. 3-49. [2] Зорич А. В. // УМН. 1984. Т. 39. № 5. С. 235-236. [3] Дынников И. А. // Матем. заметки. 1993. Т. 53. № 5. С. 57-68. [4] Novikov S. P. // Geom. Funct. Anal. 1995. V. 5. № 2. Р. 434-444. [5] Новиков С. П., Мальцев А.Я. // УФН. 1998. Т. 41. № 3. С. 231-239. [6] Дынников И. А. // УМН. 1999. T. 54. № 1. C. 21-60. [7] De Leo R. // Preprint. University of Maryland at College Park. [8] Alligood K. T., Sauer T. D., Yorke J. A. Chaos. An Introduction to Dynamical Systems. New York: Springer-Verlag, 1996. 